\section{Shelter from the}

\section{mental storm}

$\mathrm{H}$ omeless people are more likely to suffer from a mental illness than the general population, and to be admitted to hospital as a result, says a new report by the Canadian Institute for Health Information.

The report, Improving the Health of Canadians: Mental Health and Homelessness, paints a portrait of Canada's estimated Io 000 homeless as a population for which the emergency room is often a major source of help.

In 2005/2006, mental illness diagnoses accounted for $52 \%$ of admissions of homeless people to acute care hospitals, compared with pregnancy and childbirth as the most frequent reason for admissions to hospital among other patients ( $13 \%$ ).

Mental disorders also prompted $35 \%$ of the visits of homeless people to emergency departments, even if they did not end up being admitted. By contrast, injuries and poisonings were the most common reasons for emergency visits by other, more well-to-do patients (25\%).

The link between mental illness, homelessness and poverty in general is complex, says Dr. Jennifer Zelmer, vice-president for research and analysis at the Canadian Institute for Health Information, and a co-author of the report. It's not clear whether homelessness increases a person's likelihood of becoming mentally ill, or whether poverty in general and homelessness specifically result from mental illness, in some cases.

Compromised mental health and mental illness can be both a risk for and an outcome of homelessness, the report points out.

"It's probably not as simple as it's always one pathway or another - it's probably a bit of both," Zelmer told $C M A J$ in an interview.

"In some cases it may well be that a severe mental illness leads to loss of employment and that's the trigger factor for ending up being homeless. But we also know that homeless youth tend to report much higher stress levels than other youth, and that's one of the risk factors for mental illness."

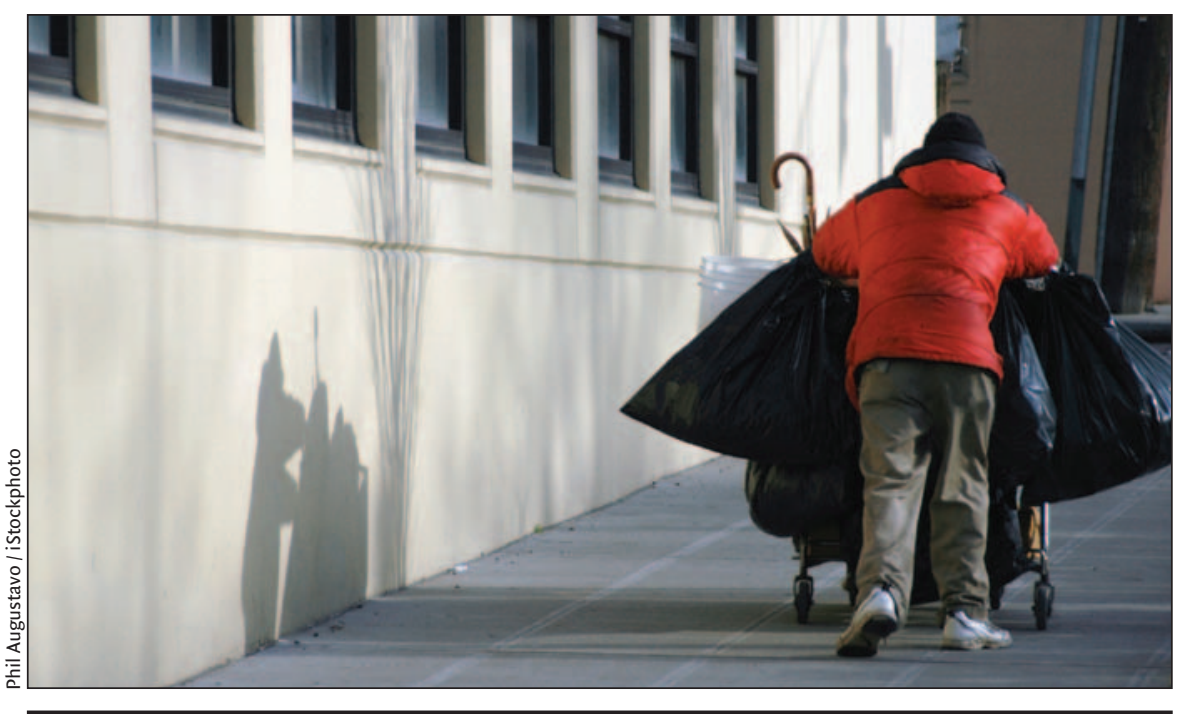

Mental illness can be both a risk for and an outcome of homelessness, according to a new Canadian Institute for Health Information report.

Poverty, inadequate income and spending an excessive amount of one's income on shelter are all risk factors for mental illness, Zelmer says.

In addition, "we do know that some types of mental illness are more common among people who have lower incomes," she added.

People living on the street are much more likely to report mental illnesses, including schizophrenia and depression, and that is reflected in their use of health services, she says. They are also less likely to report healthy coping strategies, another risk factor for mental illness.

Several studies cited in the report identify higher stress and substance abuse among street youth compared with the general population, as well as higher incidence of self-harm and suicide attempts.

One national survey, for the Public Health Agency of Canada, reported 2\% of males and $6 \%$ of females aged $15-25$ attempted suicide in Canada. A 2006 British Columbia survey, however, indicates $15 \%$ of males and $30 \%$ of females living on the street or identifying as "marginalized" had attempted suicide at least once in the previous year.

Although the report does not make recommendations, it provides examples of the type of programs that appear to help, such as housing first programs and assertive community treatment approaches. The housing first model, such as New York City's "Pathways to
Housing," focuses on providing people with shelter and then offering services to treat addictions or mental illness. It has been shown to be effective in reducing homelessness and the amount of time that people with mental illness spend in hospital, says Zelmer.

Assertive community treatment programs, such as the Psychiatric Outreach Team of the Royal Ottawa Hospital, focus on taking help to people, rather than having them come to more traditional treatment settings.

"Findings indicate that there may be value in clinical, outreach and research programs that target specific issues, such as coping skills, self-worth and social support, along with interventions and policies that target mental illness, addictions and other determinants of homelessness such as housing, income and employment," the report says.

This report was the first to compile and examine homeless people's use of hospitals, because it has only been since 2005 that hospitals have identified homeless patients in their records. Health care workers now have a special postal code to identify people with no fixed address.

Before 2005, "there was no way of identifying or understanding what the health problems were for the homeless, compared to others," says Zelmer. — Laura Eggertson, Ottawa

DOI:Io.1503/cmaj.071360 